\title{
In Vivo Photoacoustic Imaging of Blood Vessels Using an Extreme-Narrow Aperture Sensor
}

\author{
Roy G. M. Kolkman, Erwin Hondebrink, Wiendelt Steenbergen, and Frits F. M. de Mul
}

\begin{abstract}
We applied photoacoustics for noninvasive two-dimensional imaging of blood vessels in vivo, using near infrared light. This study was undertaken to develop photoacoustic tomography of tissue for the detection of embedded blood vessels using a newly developed piezoelectric double ring detector, featuring an extremely narrow aperture.
\end{abstract}

Index Terms-Biomedical imaging, blood, photoacoustic effects, piezoelectric devices, tomography.

\section{INTRODUCTION}

I IT IS OUR purpose to show the feasibility of photoacoustic imaging (PAI) to detect blood vessels hidden in tissue in a noninvasive way. In PAI, the tissue is illuminated by a short light pulse (about $10 \mathrm{~ns}$ ), which is partly absorbed at positions where absorbing particles, like red blood cells, are present. A local adiabatic temperature rise will occur, followed by a pressure rise due to restricted dilatation. This pressure wave will propagate through the tissue and, thus, can be detected at the surface. Here, we present what are to our knowledge the first in vivo photoacoustic (PA) cross-sectional images of single human blood vessels.

With all optical visualization techniques of subsurface structures in highly scattering media, like dermal tissue, the large scattering coefficient limits not only the penetration of light into the medium, but also hampers the possibilities to localize optical absorbers with satisfactory resolution. The penetration depth of the light is limited to about $1 \mathrm{~cm}$ at $550 \mathrm{~nm}$ and to several centimeters at $850 \mathrm{~nm}$ and $1064 \mathrm{~nm}$.

With photoacoustic signal generation, however, the signal amplitude is determined by the local fluence rate only. In this way, blood vessels or tumors may be imaged in vivo by choosing the appropriate wavelength for absorption and measuring the pressure in a plane of detection at the surface as a function of detector position and ultrasound time-of-flight. It was shown [1]-[4] that the PAI technique makes it possible to image structures with $20-\mu \mathrm{m}$ depth resolution. The PA-sensitivity depends on the contrast in absorption by blood with respect to the surrounding medium, which is a factor of about 100 at $550 \mathrm{~nm}$ to about 10 at $850 \mathrm{~nm}$.

Apart from high-resolution imaging, PA has been used in imaging of layered tissue structures [12]-[15], or to localize

Manuscript received October 10, 2002; revised February 6, 2003. This work was supported in part by the Technology Foundation of the Netherlands STW under Grant TTN.4661

The authors are with the Faculty of Science and Technology, University of Twente, AE Enschede NL-7500, The Netherlands (e-mail: r.g.m.kolkman@ utwente.nl).

Digital Object Identifier 10.1109/JSTQE.2003.813302 absorbing objects in tissue for applications like mammography [16], [17].

Photoacoustic signal generation can also be used to determine the optical properties of turbid media such as tissue [5], [6]. Based on changes in these optical properties, systems are being developed to detect changes in concentration of tissue chromophores such as hemoglobin [7]-[9] (i.e., oxygenation) or glucose [10], [11].

\section{MATERIALS AND MethodS}

To generate photoacoustic signals, a pulsed light source is required. If this light source generates pulses with a pulse duration shorter than the acoustic transit time $(=\delta / \nu)$ of the acoustic source ( $\nu$ is the acoustic velocity, $\delta$ is the optical penetration depth, assuming that the beam diameter is much larger than $\delta$ ) the condition of stress confinement is fulfilled. In this case, the initial pressure distribution is proportional to the deposited energy density [18]. We used a $Q$-switched Nd: YAG laser, which generates light pulses of $1064 \mathrm{~nm}$ (first harmonic) with a duration of $10 \mathrm{~ns}$, and a repetition rate of $10 \mathrm{~Hz}$ (Quanta Ray DCR-2, Spectra Physics). The pulse duration fulfils the requirements of stress confinement, when using photoacoustics to localize blood in the visible and near-infrared wavelength range. The light is coupled into a glass fiber, which is integrated in the photoacoustic sensor. A schematic overview of the setup is shown in Fig. 1.

The home-built double ring sensor consists of two concentric ring-shaped electrodes [19]. The inner ring has an inner radius of $2 \mathrm{~mm}$ and a width of $0.17 \mathrm{~mm}$. The outer ring has an inner radius of $3.5 \mathrm{~mm}$ and a width of $0.1 \mathrm{~mm}$, so that the area of both rings is equal. The piezoelectric material $(25-\mu \mathrm{m}$-thick PVdF, biaxially stretched, electrically polarized, with one side metallized $\mathrm{Au} / \mathrm{Pt}$, Piezotech SA, France) is glued to the electrodes using significant pressure to minimize the thickness of the glue layer. The two ring shaped electrodes are connected to amplifiers. The sensor is embedded in a brass housing to shield the electronics for electromagnetic noise. An optical fiber (core diameter $600 \mu \mathrm{m}, \mathrm{NA}=0.22$ ) is placed in the center of the sensor to deliver light pulses to the tissue. A schematic drawing of the cross section of the sensor is shown in Fig. 2. This sensor has an aperture of $3^{\circ}(-6 \mathrm{~dB}$ of directivity function) for acoustic sources with a peak-to-peak time of $100 \mathrm{~ns}$. To be able to scan over the tissue surface, the sensor is mounted in an XYZ scanning system.

Photoacoustic signals were generated by illuminating the tissue through a fiber, which was positioned in the center of the photoacoustic sensor. The gap between the sensor and the 


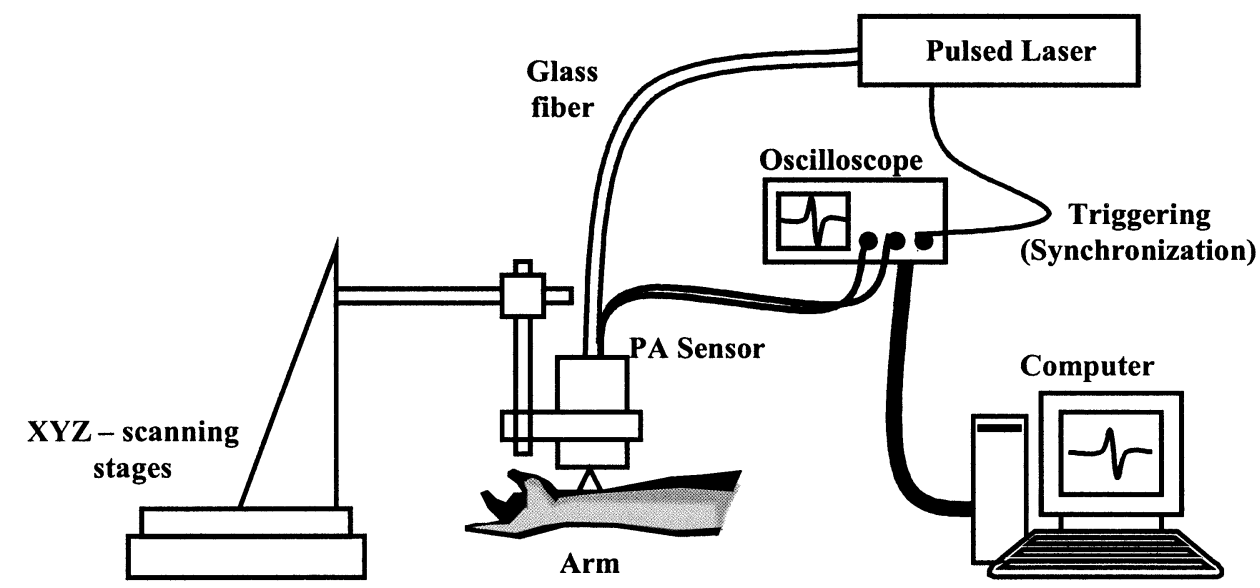

Fig. 1. Setup for photoacoustic imaging in the human arm.

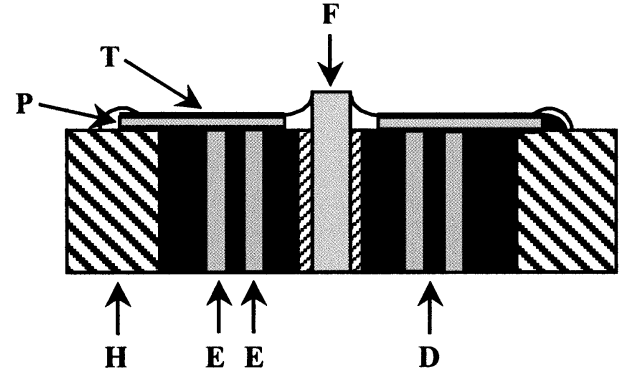

Fig. 2. Cross section of the double ring photoacoustic sensor. This sensor has two concentric ring shaped sensor surfaces. F: Optical fiber $(600-\mu \mathrm{m}$ diameter). P: Piezo film (thickness $25 \mu \mathrm{m}$ ). T: Au top layer of piezo film, grounded. H: Housing, grounded. E: Ring shaped electrodes, diameters $4.00 \times$ 4.34 and $7.00 \times 7.20 \mathrm{~mm}$. D: Dielectric.

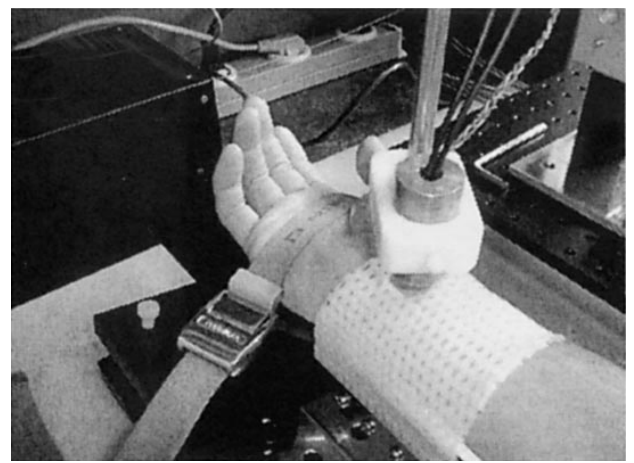

Fig. 3. Position of arm during scanning of the branching of two veins in the lower side of the wrist. To keep the wrist in a stable position, a mould of a thermoplastic sheet material (Efficast, Orfit Industries) has been used.

skin surface was filled with ultrasound contact gel (Sonogel, Germany) to ensure a good optical and acoustical coupling. The energy applied to the tissue was $1 \mathrm{~mJ} / \mathrm{pulse}$. An arm support has been used to keep the arm in a stable position during the measurement, as shown in Fig. 3. To keep the wrist in fixed position, a mould made of a thermoplastic sheet material (Efficast, Orfit Industries) has been used.

The expected photoacoustic signals contain frequencies in the range from about $1-30 \mathrm{MHz}$, corresponding to blood vessels with diameters from $1 \mathrm{~mm}$ down to $0.03 \mathrm{~mm}$. Therefore, our sampling rate has to be at least $60 \mathrm{Msamples} / \mathrm{s}$ to avoid aliasing. In our setup, a dual channel, digital oscilloscope (TDS220 - Tektronix, 100-MHz bandwidth) has been used. It was set to acquire photoacoustic signals at a sampling rate of $250 \mathrm{Msamples} / \mathrm{s}$. The acquisition is synchronized (triggered) on the $Q$-switch trigger, generated by the laser. The delay between the $Q$-switch trigger and the actual light pulse, exiting the fiber inside the photoacoustic sensor, is corrected by applying a delay to the $Q$-switch trigger pulse, before it synchronizes (triggers) the oscilloscope.

The photoacoustic time traces at each measurement position were converted to depth information by multiplying the time with the velocity of sound in tissue $(1500 \mathrm{~m} / \mathrm{s})$. Next, we assumed that we could approach the measured photoacoustic signals by the signal of a photoacoustic point source as described by Sigrist et al. [20] and Hoelen et al. [2]. This bipolar signal (derivative of a Gaussian) was fitted to our measured time trace. The resulting fit was integrated over depth (time) to obtain the absorption distribution. This absorption distribution corresponds with the cross-section of the blood vessel.

Imaging with the double ring sensor can be carried out by scanning the sensor over the tissue surface, which is comparable to an echographic B-scan. As the opening angle of the sensor is very small (about $3^{\circ}[19]$ ), the time traces can be regarded as a one-dimensional (1-D) depth image of photoacoustic sources inside the measurement volume. When a linear scan is made, a two-dimensional (2-D) image (scan direction versus depth) is obtained by plotting all the 1-D depth images (i.e., time traces) next to each other in a 2-D image plane.

Our approach as described above, is different from three-dimensional (3-D) high-resolution imaging as published by Hoelen et al. [1]-[4] or tomographic imaging. This uses reconstruction algorithms which make use of the fact that signals from a single source are detected at multiple sensor positions, improving the signal to noise ratio by using signals from multiple sensor positions to reconstruct the image. This requires overlapping sensor apertures. Furthermore, all data should be available before image reconstruction can be carried out; the reconstruction is off-line. In our approach, we use a sensor with an extremely narrow aperture. This allows us to treat all measurements as individual 1-D depth profiles. This permits real-time reconstruction of the corresponding part of the image, without the need of the data of the adjacent elements. To enhance the signal to noise ratio in our images, we have averaged 16 photoacoustic time traces at each measurement position. 

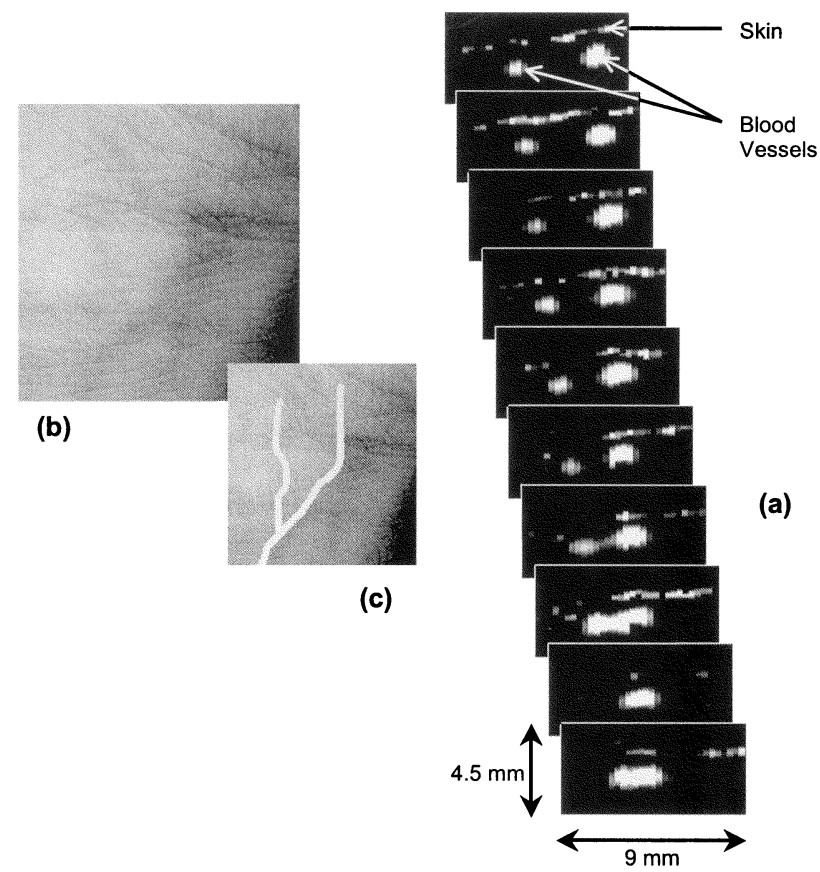

Fig. 4. Imaging of two joining veins proximal to the wrist of a human volunteer. (a) Photoacoustic image, consisting of ten slices, 1-mm apart. Horizontal axis: Scan direction [mm]; Vertical axis: Depth [mm]. Each image consists of 61 measurement positions along a line, with a separation of $0.3 \mathrm{~mm}$ At each measurement position, the photoacoustic signals are averaged 16 times. (b), (c) Photograph of measurement site; in (c) the course of the vessels is indicated with the white line.

To avoid lateral broadening (in scan direction) of the vessels in the image, we used the zero-time-shift cross-correlation value of the two signals of the separate ring-shaped electrodes as weight factors in the image. Each electrode's time-trace (1-D depth image) is multiplied by its weight factor. The cross-correlation value will be maximum when the photoacoustic source (blood vessel) is located exactly on the midline of the sensor, as in this case both ring-shaped electrodes receive photoacoustic signals with identical shape. When the source is located off-axis, the shape of the signals detected by the two electrodes will not be equal. This is caused by different interference at the ringshaped sensor surfaces due to the different dimensions (radii) of the two rings. Thus, the cross-correlation value decreases, diminishing the weight factors and weakening the images of these off-axis sources. By this, the sensor's aperture is further reduced which suppresses the lateral broadening of the vessel image.

\section{RESUlTS AND DisCUSSION}

The measurements consisted of nine linear scans, with a spacing of $1 \mathrm{~mm}$ between each scan line, along the arm direction. Each scan line itself consisted of 31 measurement positions, with a spacing of $0.3 \mathrm{~mm}$. At each measurement position the photoacoustic time traces were measured. This approach provided a 2-D image (in depth and laterally) for each scan line. At each measurement position, the time traces were averaged 16 times to enhance the signal to noise ratio. The total scan time was 20 min.

In Fig. 4, the photoacoustic image of two joining superficial palmar veins and the downstream median vein, a few centime- ters proximal to the wrist is shown. A photograph of the scanned area is shown in the left panel. The photoacoustic reconstruction is presented in the right panel. Besides the blood vessels, the skin produces a photoacoustic signal, as due to the difference in optical absorption between the ultrasound contact gel (used as acoustic coupling medium between the skin and the sensor) and the skin. This figure shows that the cross section of blood vessels in the skin can be imaged with high contrast, showing even the joining of the vessels. Visual inspection (photograph-left panel) shows a 2-D image, in which vaguely a blurred image of the vessels can be seen.

The cross-sectional images show the shape of the blood vessels to be oval. The smaller vessel (vessel on the left side in the photoacoustic image) has a size in depth of about $0.6 \mathrm{~mm}$, and the larger vessel on the right side has a size in depth of $1 \mathrm{~mm}$. The depth position of the vessels with respect to the skin surface is about $1 \mathrm{~mm}$.

Visual inspection through the skin gives a size of the vessels that is slightly larger than estimated with our photoacoustic setup. This is caused by light scattering in the skin, which causes these vessels only to be visible vaguely.

\section{CONCLUSION}

We demonstrated in vivo 2-D photoacoustic imaging of individual human blood vessels using a photoacoustic sensor with an extremely narrow opening angle. The joining of two palmar veins proximal to the wrist is reconstructed, showing the size of the vessel, as well as the depth position of the vessels with respect to the skin surface. The extremely narrow opening angle of the sensor allows us to construct a photoacoustic image without recourse to any image reconstruction algorithms.

These first in vivo photoacoustic images of single human blood vessels, offer new perspectives for application in various clinical fields, like in oncology by measuring angiogenesis, or in imaging of the micro and macrovascular system.

\section{REFERENCES}

[1] C. G. A. Hoelen, F. F. M. de Mul, R. Pongers, and A. Dekker, "Three-dimensional photoacoustic imaging of blood vessels in tissue," Opt. Lett., vol. 23, pp. 648-650, 1998.

[2] C. G. A. Hoelen and F. F. M. de Mul, "A new theoretical approach to photoacoustic signal generation," J. Acoust. Soc. Amer, vol. 106, pp. 695-706, 1999.

[3] C. G. A. Hoelen, A. Dekker, and F. F. M. de Mul, "Detection of photoacoustic transients originating from microstructures in optically diffuse media such as biological tissue," IEEE Trans. Ultrason., Ferroelect. Freq. Contr., vol. 48, pp. 37-47, 2001.

[4] C. G. A. Hoelen and F. F. M. de Mul, "Image reconstruction for photoacoustic scanning of tissue structures," Appl. Opt., vol. 39, pp. $5872-5883,2000$.

[5] A. A. Oraevsky, S. L. Jacques, and F. K. Tittel, "Measurement of tissue optical properties by time-resolved detection of laser-induced transient stress," Appl. Opt., vol. 36, pp. 402-415, 1997.

[6] A. A. Karabutov, N. B. Podymova, I. M. Pelivanov, S. E. Skipetrov, and A. A. Oraevsky, "Direct measurement of axial distribution of absorbed optical energy in turbid media by time-resolved optoacoustic method," Proc. SPIE, vol. 3916, pp. 112-121, 2000.

[7] R. Fainchtein, B. J. Stoyanov, J. C. Murphy, D. A. Wilson, and D. F. Hanley, "Local determination of hemoglobin concentration and degree of oxygenation in tissue by pulsed photoacoustic spectroscopy," in Proc. SPIE, vol. 3916, 2000, pp. 19-33.

[8] - "Invivo photoacoustic spectroscopy of hemoglobin in cerebral tissue," Prog. Nat. Sci., vol. 6, pp. S589-S593, 1996. 
[9] G. Paltauf, K. P. Köstli, D. Frauchiger, and M. Frenz, "Spectral optoacoustic imaging using a scanning transducer," Proc. SPIE, vol. 4434, pp. 81-88, 2001

[10] A. Bednov, A. Karabutov, E. Savateeva, W. March, and A. Oraevsky, "Glucose monitoring in vivo by measuring laser-induced acoustic profiles," Proc. SPIE, vol. 3916, pp. 9-18, 2000.

[11] Z. Zhao and R. Myllyla, "Photoacoustic determination of glucose concentration in whole blood by a near-infrared laser diode," Proc. SPIE, vol. 4256, pp. 77-83, 2001.

[12] G. Paltauf, K. P. Köstli, D. Frauchiger, and M. Frenz, "Spectral optoacoustic imaging using a scanning transducer," Proc. SPIE, vol. 4434, pp. 81-88, 2001.

[13] G. Paltauf and H. Schmidt-Kloiber, "Pulsed optoacoustic characterization of layered media," J. Appl. Phys., vol. 88, pp. 1624-1631, 2000.

[14] K. P. Köstli, M. Frenz, H. P. Weber, G. Paltauf, and H. Schmidt-Kloiber, "Pulsed optoacoustic tomography of soft tissue with a piezoelectric ring sensor," Proc. SPIE, vol. 3916, pp. 67-74, 2000.

[15] A. A. Karabutov, E. V. Savateeva, and A. A. Oraevsky, "Imaging of layered structures in biological tissues with opto-acoustic front surface transducer," Proc. SPIE, vol. 3601, pp. 284-296, 1999.

[16] A. A. Oraevsky, A. A. Karabutov, S. V. Solomatin, E. V. Savateeva, V. G. Andreev, Z. Gatalica, H. Singh, and R. D. Fleming, "Laser optoacoustic imaging of breast cancer in vivo," Proc. SPIE, vol. 4256, pp. 6-15, 2001.

[17] A. A. Karabutov, V. G. Andreev, B. Bell, R. D. Fleming, Z. Gatalica, M. Motamedi, E. V. Savateeva, H. Singh, S. V. Solomatin, S. L. Thomsen, P. M. Henrichs, and A. A. Oraevsky, "Optoacoustic images of early cancer in forward and backward modes," Proc. SPIE, vol. 4434, pp. 13-27, 2001.

[18] K. P. Köstli, M. Frenz, H. P. Weber, G. Paltauf, and H. Schmidt-Kloiber, "Optoacoustic infrared spectroscopy of soft tissue," J. Appl. Phys., vol. 88, pp. 1632-1637, 2000

[19] R. G. M. Kolkman, M. C. Pilatou, E. Hondebrink, and F. F. M. de Mul, "Photo-acoustic A-scanning and monitoring of blood content in tissue," Proc. SPIE, vol. 3916, pp. 76-83, 2000.

[20] M. W. Sigrist and F. K. Kneubühl, "Laser generated stress waves in liquids," J. Acoust. Soc. Amer., vol. 64, pp. 1652-1663, 1978.
Roy G. M. Kolkman was born in Lichtenvoorde, The Netherlands, in 1974. He received the M.Sc. degree in applied physics from the University of Twente, Enschede, The Netherlands, in 1998. From October 1998 to October 2002, he worked toward the Ph.D. degree in the biophysical engineering group in the Department of Applied Physics, University of Twente.

Currently, he is a Postdoctoral Researcher in the biophysical engineering group. His research interests include photoacoustic imaging and laster Doppler flowmetry.

Erwin Hondebrink was born in Enschede, The Netherlands, in 1974. He received the B.Sc. degree in electrical engineering from the Hogeschool Enschede, Enschede, The Netherlands, in 1998.

$\mathrm{He}$ is currently working as an Electrical Engineer in the Biophysical Engineering Group in the Department of Applied Physics, University of Twente, Enschede, The Netherlands. His current research interests include photoacoustic imaging and laser Doppler flowmetry.

Wiendelt Steenbergen was born in 1964 in Meppel, The Netherlands. He received the Master's degree in aerospace engineering from Delft University of Technology, Delft, The Netherlands, where he specialized in laser Doppler anemometry and low-speed aerodynamics. His $\mathrm{Ph} . \mathrm{D}$. research at the Eindhoven University of Technology, Eindhoven The Netherlands, focused on turbulent pipe flows

Currently, he is Assistant Professor in biomedical optics at the University of Twente, Enschede, The Netherlands, working on optical techniques for medical diagnosis.

Frits F. M. de Mul received the M.Sc. and Ph.D. degrees in radiation physics study of neutron scattering from the Technical University, Delft, The Netherlands, in 1973 .

After three years of teaching at secondary school level, he joined the Department Applied Physics, University Twente, Enschede, The Netherlands, where he holds a combined position of teaching physics and R\&D in biomedical optics. 